\title{
Strange Attractor in Density Evolution
}

\author{
Sinan Kahraman \\ Department of Electrical-Electronics Engineering \\ Bilkent University \\ Ankara, TR-06800, Turkey \\ Email: sinank@ieee.org
}

\begin{abstract}
The strange attractor represents a complex pattern of behavior in dynamic systems. This paper introduces a strange attractor of channel polarization as a result of a geometric property of density evolution for code construction. In this way, we can define a subset of synthetic channels that are universally (channel independently) less reliable than the original channel. We show that the cardinality of the attractor set is the $n+2$-th Fibonacci number for the block length $N=2^{n}$. This can be accepted that it is a significantly large number for very long codes. Recently, it is known that polar codes can be constructed with sub-linear complexity by the use of partial orderings. In this study, we additionally define $1+\log _{2} \log _{2} N$ universal operators. Finally, we show that these universal operators can be applied on the attractor set to increase the number of synthetic channels that are universally less reliable than the natural channel.

Index Terms-Channel polarization, partial orders, strange attractor.
\end{abstract}

\section{INTRODUCTION}

Polar coding is the first and only coding technique to provably achieve the channel capacity for binary discrete memoryless channels using quasi-linear complexity encoding, decoding and code construction methods defined in detail [1]. This technique received great interest due to this important advantage. It has been discussed in 3GPP standardization works and accepted to be used in 5G technology. From an industry point of view, this result demonstrates that polar coding can be considered for use in different technologies where long code lengths are preferred to achieve higher reliabilities for a fixed code rate (i.e., size of information set divided by code length.) This paper aims to design very long polar codes.

In polar coding, conventional code construction is defined as determination of the order of reliabilities of all synthetic channels. Using Monte-Carlo simulation is a way for the code construction described in [1]. Later, since polar coding is a channel specific technique, the code construction problem for polar codes has been studied as a research topic in the literature. This is mainly due to the fact that once the polar code is designed for communication systems, it is necessary to make this design specific to the channel. For this reason, various construction methods based on calculating the reliability of the synthetic channels are discussed by density evolution [2], upgrading and downgrading [3], and Gaussian approximation [4]. A comperative study in [5] investigates the performance of these polar code constructions.

Recently in [6] and [7], a partial order for synthetic channels is defined as an universal (channel independent) property of the channel polarization. This feature has been considered in [8] to reduce the complexity of polar code design based on the considered calculations. As a result, it has been shown in [8] that code design for polar codes can be done with very low complexity such as a sub-linear complexity.

\section{A. System Model and Preliminary Details}

The structure of the polar codes in [1] with the block length $N$ is introduced by $G=F^{\otimes n}$ matrix. It is defined by the $n^{t h}$ Kronecker power of $2 \times 2$ kernel matrix $F$. The encoding task is expressed as $x=u G$, in modulo- 2 arithmetic. Polar coding in [1] has low complexity encoder and decoder. Here, FFT-like structures require $\mathcal{O}(N \log N)$ complexity. The input vector $u$ with $N$ length contains $K$ information components and $N-K$ frozen components for the coding rate $R=\frac{K}{N}$. It is assumed that the frozen locations are known by the receiver. Here, $N-K$ synthetic channels that are the lowest reliable are reserved for frozen components. High reliability is considered as large mutual information, small Bhattacharya parameter and small error probability. Code design methods provides the locations of frozen components for polar coding. The transition probabilities of the synthetic channels obtained after one-step of polarization are defined as follows:

$$
\begin{aligned}
W^{-} & =W\left(y_{1}, y_{2} \mid u_{1}\right) \\
& =\frac{1}{2} \sum_{u_{2}=0}^{1} W\left(y_{1} \mid u_{1} \oplus u_{2}\right) W\left(y_{2} \mid u_{2}\right), \\
W^{+} & =W\left(y_{1}, y_{2}, u_{1} \mid u_{2}\right) \\
& =\frac{1}{2} W\left(y_{1} \mid u_{1} \oplus u_{2}\right) W\left(y_{2} \mid u_{2}\right),
\end{aligned}
$$

where $y_{1}$ and $y_{2}$ are noisy observation of the receiver unit, $u_{1}$ and $u_{2}$ are the input of one-step polarization. Here, $W^{-}$ denotes the polarized bad channel. $W^{+}$is the polarized good channel. $W^{+}$has higher reliability than $W^{-}$and this is represented as $W^{-} \prec W \prec W^{+}$, where $W$ is the natural channel. Reliability ordering for $N$ synthetic channels depends on the natural channel. For that reason, code designs are channel specific that are based on Monte-Carlo simulation or density evolution calculation by the use of Gaussian approximation. The solution to the problem is sufficient to be done only once. Unfortunately, it is channel specific that is the major issue of the code design. Recent researches on the partial order have focused on this problem. They exploited relative non-channel specific solutions of the synthetic channels. 
The following definitions were provided for this purpose. Any synthetic channel such as $\left(\cdots\left(\left(W^{+}\right)^{-}\right)^{+} \cdots\right)^{-}=$ $W^{+-+\cdots-}$ obtained by $n$-step polarization is mapped to index in $[0, N)$ using 1 for + and 0 for - polarization step. E.g., $W^{--++}: W_{3}$ with $(0011)$ binary index and $W^{+--+}: W_{9}$ with (1001) binary index.

Let $k_{i}$ be the $i^{t h}$ most significant bit of the binary index of $k$.

Definition 1 (The first order operator). Addition.

If $k_{i}=1$ and $k_{j}=\ell_{j}$ for all $j$ where $j \neq i$, then $W_{\ell} \preceq W_{k}$.

Definition 2 (The second order operator). Left swap.

If $k_{i}, k_{i+t}=10$ and $\ell_{i}, \ell_{i+t}=01$ and also $k_{j}=\ell_{j}$ for all $j$ and $t \geq 1$ where $j \neq i$ and $j \neq i+t$, then $W_{\ell} \prec W_{k}$.

For more clarity, we have the results $W_{(a b 0 c)} \prec W_{(a b 1 c)}$ and $W_{(a 01 b)} \prec W_{(a 10 b)}$. This partial order provides a sublinear complexity code design in [8] and [9].

\section{B. A New Partial Order}

In this section, we introduce a new way for partial order to reduce the complexity of the code design in [9]. For this purpose, we first define a feature of partial order by using multiple operators. It has been shown that the proposed new feature can sort synthetic channels with a small difference in reliability that can not be separated by the known partial order with the Definition 1 and 2. It can be noticed that these orderings are still universal. To introduce the multiple partial order, we define new operators as follows:

Definition 3 (A new partial order). Multiple.

For a given universal partial order $W_{\ldots 0} \ldots \prec W \ldots 1 \ldots$ (the first order operator), it is easy to notice that $W_{\ldots 01 \ldots} \prec$ $W \ldots 10 \ldots$ (the second order operator) is also universal partial order. Recursively, $W_{\ldots 0110 \ldots} \prec W_{\ldots 1001 \ldots}$ and $W_{\ldots 01101001 \ldots} \prec W \ldots 10010110 \ldots$ are universal partial orders.

This property is a natural extension of the left swap operator in Definition 2. The number of operators that can be given for the block length $N$ is $1+\log _{2} \log _{2} N$. Here, we provide 5 operators that are given in Table I for $N=65536$. This result shows that the multiple partial order relations can provide more than two operators for $N \geq 16$.

Following examples can be given as a result of the new feature.

Example 1. By using $3^{\text {rd }}$ order operation,

$$
W_{(0110)} \prec W_{(1001)} \text {. }
$$

Example 2. By using $4^{\text {rd }}$ order operation,

$$
W_{(01101001)} \prec W_{(10010110)} \cdot
$$

They are universal partial orders that can not be obtained by the Definitions 1 and 2. Notice that the resolution of the new operators are higher than the previous definitions. This is an important property that can be exploited to order antichains.
TABLE I

MULTIPLE OPERATORS FOR $N=65536$

\begin{tabular}{|c|c|}
\hline order & operator (less reliable $\prec$ more reliable) \\
\hline $1^{\text {st }}$ & $0 \prec 1$ \\
\hline $2^{\text {nd }}$ & $01 \prec 10$ \\
\hline $3^{\text {rd }}$ & $0110 \prec 1001$ \\
\hline $4^{t h}$ & $01101001 \prec 10010110$ \\
\hline $5^{\text {th }}$ & $0110100110010110 \prec 1001011001101001$ \\
\hline
\end{tabular}

\section{Gaussian Approximation for Density Evolution}

The Gaussian approximation for density evolution was first proposed by Chung et al. to analyze low density parity check codes in [10]. Then, the Gaussian approximation was used by Trifonov in [4] as one of the deterministic ways to compute the reliability of synthetic channels. We summarized this method as the following way.

We assume that the all-zero codeword is transmitted to the receiver. The log-likelihood ratio (LLR) for a noisy observation $y_{i}=x_{i}+n_{i}$ is defined as $L_{1}^{i}\left(y_{i}\right)=\log \frac{W\left(y_{i} \mid 0\right)}{W\left(y_{i} \mid 1\right)}$. The probability density function is $f(x)=e^{-x^{2} / 2 \sigma^{2}}$ for additive white Gaussian noise with $N\left(0, \sigma^{2}\right)$ distribution. The expected value of $L_{1}^{i}\left(y_{i}\right)$ can be considered as follows:

$$
E\left[L_{1}^{i}\left(y_{i}\right)\right]=2 / \sigma^{2} .
$$

Variance of the LLR is given as:

$$
V\left[L_{1}^{i}\left(y_{i}\right)\right]=\frac{4}{\sigma^{2}} .
$$

The update rules for the expectations of inter-level LLRs is given for $i=1, \ldots, n / 2$ as follows:

$$
\begin{aligned}
E\left[L_{j}^{(2 i-1)}\right] & =\phi^{-1}\left(1-\left(1-\phi\left(E\left[L_{j / 2}^{i}\right]\right)\right)^{2}\right), \\
E\left[L_{j}^{(2 i)}\right] & =2 E\left[L_{j / 2}^{i}\right]
\end{aligned}
$$

where

$$
\phi(x)=\left\{\begin{array}{ll}
1-\frac{1}{\sqrt{4 \pi x}} \int_{-\infty}^{\infty} \tanh \frac{u}{2} e^{-\frac{(u-x)^{2}}{4 x}} d u & x>0 \\
1, & x=0
\end{array} .\right.
$$

The error probability of indices $i \in\{1, \ldots, N\}$ is given as follows:

$$
\pi_{i} \approx Q\left(\sqrt{E\left[L_{N}^{i}\right] / 2}\right)=\frac{1}{2} \operatorname{erfc}\left(\frac{1}{2} \sqrt{E\left[L_{N}^{i}\right]}\right)
$$

where

$$
\operatorname{erfc}(x)=\frac{2}{\sqrt{\pi}} \int_{x}^{\infty} e^{-v^{2}} d v .
$$

An upper bound of the error probability is the sum of error probabilities for the set of information indices.

To simplify the update rule we use an approximation of

$$
\tanh x \approx\left\{\begin{array}{cc}
1, & x>0 \\
0, & x=0 \\
-1, & x<0
\end{array}\right.
$$


as given in Appendix I, and hence, the simplified update rule can be provided by using the following definitions:

$$
\begin{aligned}
\phi(x) & =\operatorname{erfc}\left(\frac{\sqrt{x}}{2}\right) \\
\phi^{-1}(x) & =4(\operatorname{erfcinv}(x))^{2}
\end{aligned}
$$

The simplified update rule is given as follows:

$$
\begin{gathered}
E\left[L_{j}^{(2 i-1)}\right]= \\
4\left(\operatorname{erfcinv}\left(1-\left(1-\operatorname{erfc}\left(\frac{1}{2} \sqrt{E\left[L_{j / 2}^{i}\right]}\right)\right)^{2}\right)\right)^{2} \\
E\left[L_{j}^{(2 i)}\right]=2 E\left[L_{j / 2}^{i}\right] .
\end{gathered}
$$

This is a numerically stable method that can be efficiently implemented by only using a lookup table for the function $\operatorname{erfc}(\mathrm{x})$ and $\operatorname{erfcinv}(\mathrm{x})$.

\section{THE STRANGE ATtRACTOR}

In the previous section, the reliability of synthetic channels was computed by using the simplified Gaussian approximation update functions. In this section, we investigate the behaviors of these update functions by using the geometric properties. First, $y=2 x$ and $y=\phi^{-1}\left(1-(1-\phi(x))^{2}\right)$ functions are depicted in Fig. 1. Moreover, the reflections of these curves with respect to the $y=x$ line are also added.

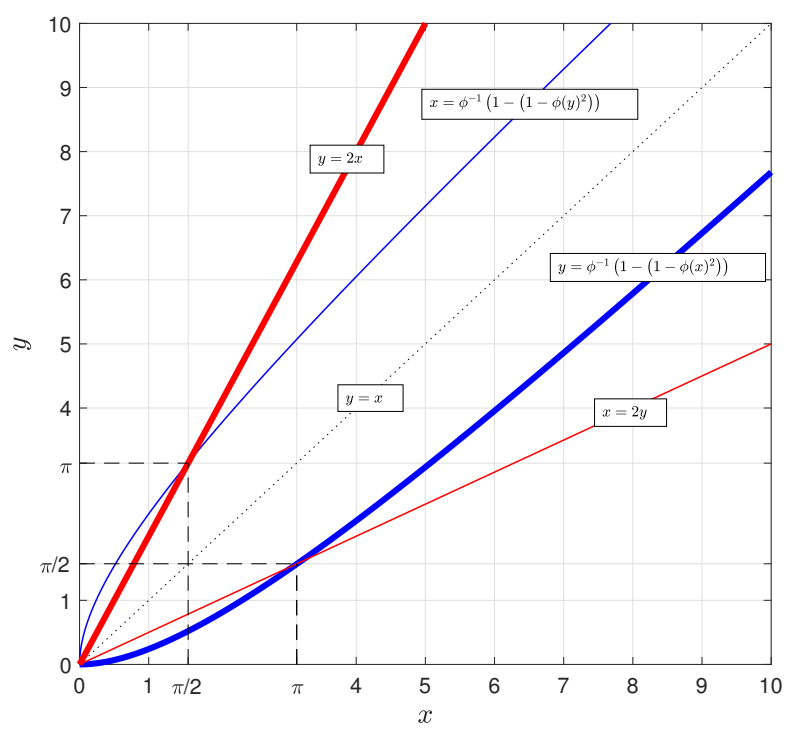

Fig. 1. Plot of the recursive functions for update rule of Gaussian approximation method. (bold curves: the functions and thin curves: the reflections.)

Here, we provide some relations of these functions as follows:

Let us define the functions $f_{1}(x)=x / 2$ and $f_{2}(x)=$ $\phi^{-1}\left(1-(1-\phi(x))^{2}\right)$.

i) $y=f_{1}(x)$ and $y=f_{2}(x)$ intersect at $(x=0, y=0)$. ii) $y=f_{1}(x)$ and $y=f_{2}(x)$ intersect at $(x=\pi, y=\pi / 2)$.

iii) $f_{1}(x)>f_{2}(x)$ for $x \in(0, \pi)$.

As a result of these observations, we can universally identify a set of some synthetic channels that are less reliable than the natural channel. As a similar approach to analyze in a chaotic systems, we introduce a strange attractor to represent a complex pattern of behavior in channel polarization. We provide the following definition for a set of some polarization scenarios that are universally converged to the same result.

Definition 4. A strange attractor is a subset of synthetic channels that are universally unreliable than the natural channel $W_{k} \prec W$.

For this purpose, any natural channel with a limited LLR can be transformed to polarized synthetic channels with the index that has no ' 11 ' in binary expansion defines a strange attractor.

It can be noticed that they are universally less reliable than the natural channel with $L L R<\pi / 2$. If the block length is long enough, then the reliability of them goes to 0 .

Example 3. For the original channel with $L L R<\frac{\pi}{2}$, all possible synthetic channels that are labelled by the indices with binary expansion $\left(k_{1}, k_{2}, \ldots, k_{n}\right)$ with $k_{i} \neq 1$ and $k_{i+1} \neq 1$ for any $i=[1, n)$ are less reliable than the original channel.

Example 4. For the original channel with $L L R<\pi$, all possible synthetic channels that are labelled by the indices with binary expansion $\left(k_{1}, k_{2}, \ldots, k_{n}\right)$ with $k_{i} \neq 1$ and $k_{i+1} \neq 1$ for any $i=[1, n)$ and $k_{1} \neq 1$ are less reliable than the original channel.

Proposition 1. As the block length increases, the LLR values of the synthetic channels that are labelled by the indices with binary expansion $\left(k_{1}, k_{2}, \ldots, k_{n}\right)$ with $k_{i} \neq 1$ and $k_{i+1} \neq 1$ for any $i=[1, n)$ converges to 0 (unreliable) and the number of these type of synthetic channels is $F_{2+n}$, where $F_{i}$ is $i^{\text {th }} \mathrm{Fi}$ bonacci number in $\{1,1,2,3,5,8,13,21,34,55,89,144, \ldots\}$ for the block length $N=2^{n}$.

Proof. The proof for the proposition is presented in two parts. The first part is concerned with the exact number of $n$-length bit strings with $k_{i} \neq 1$ and $k_{i+1} \neq 1$ for any $i=[1, n)$. Let $\mathcal{A}^{i}$ be a set of $i$-length bit strings with $k_{i} \neq 1$ and $k_{i+1} \neq 1$ for any $i=[1, n)$. The cardinality of the set $\left|\mathcal{A}^{i}\right|$ can be given as follows:

i) $\left|\mathcal{A}^{1}\right|=2$ where $\mathcal{A}^{1}:\{0,1\}$

ii) $\left|\mathcal{A}^{2}\right|=3$ where $\mathcal{A}^{2}:\{00,01,10\}$

iii) $\left|\mathcal{A}^{3}\right|=5$ where $\mathcal{A}^{3}:\{000,001,010,100,101\}$

iv) $\left|\mathcal{A}^{4}\right|=8$ where

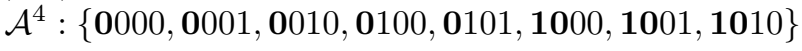

v) $\left|\mathcal{A}^{\ell}\right|=\left|\left[\mathbf{0} \mid \mathcal{A}^{\ell-1}\right],\left[\mathbf{1 0} \mid \mathcal{A}^{\ell-2}\right]\right|=\left|\mathcal{A}^{\ell-1}\right|+\left|\mathcal{A}^{\ell-2}\right|$ (Fibonacci).

As a result, $\left|\mathcal{A}^{n}\right|=F_{n+2}$ where $F_{n}=\{1,1,2,3,5,8,13,21,34,55,89,144, \ldots\}$. 
The second part of this proof is about the attractor. As a result of the observations, we could pre-define $F_{n+2}$ synthetic channels named as attractor thanks to the geometrical properties in Fig. 1 we obtained. We observe that LLR values converge to 0 for all possible bit strings with $k_{i} \neq 1$ and $k_{i+1} \neq 1$ for any $i=[1, n)$.

The number of this type of synthetic channels are provided for various number of polarization steps $n$ in Table II.

Now let's examine the asymptotic behaviour of the attractor set, which we are pre-defined. For this purpose, we provide the following expression.

$$
\lim _{N \rightarrow \infty} \frac{\text { Number of channels with (11) }}{\text { Number of all channels }}=1 .
$$

Proof is given here. We consider the expression as follows. The exact number of channels with $k_{i} \neq 1$ and $k_{i+1} \neq 1$ for any $i=[1, n)$ can be described as follows:

$$
\Delta=\Delta_{1}+\Delta_{2}
$$

where $\Delta_{1}$ is shown in Fig. 2 and $\Delta_{2}$ is shown in Fig. 3.

\begin{tabular}{|l|c|c|}
\hline $\mathcal{A}^{t}$ & 011 & all - possible \\
\hline$t$ & 3 & $n-t-3$ \\
\hline
\end{tabular}

Fig. 2. A graphical representation of the case $\Delta_{1}$.

\begin{tabular}{|l|c|}
\hline 11 & all - possible \\
\hline 2 & $n-2$
\end{tabular}

Fig. 3. A graphical representation of the case $\Delta_{2}$.

Here,

$$
\Delta_{1}=\sum_{t=0}^{n-3}\left|\mathcal{A}^{t}\right| \cdot 2^{n-t-3}
$$

and

$$
\Delta_{2}=2^{n-2} .
$$

We can show that

$$
\begin{gathered}
\Delta=2^{n-2}+\sum_{t=0}^{n-3}\left|\mathcal{A}^{t}\right| \cdot 2^{n-t-3} \\
\Delta=\left[\sum_{t=0}^{n-3} F_{t+2} \cdot 2^{n-t-3}\right]+2^{n-2} \\
=2^{n-1}\left(\left[\sum_{t=0}^{n-3} F_{t+2} / 2^{t+2}\right]+1 / 2\right) .
\end{gathered}
$$

Then, we have

$$
\Delta=2^{n-1}\left(\left[\frac{F_{2}}{2^{2}}+\frac{F_{3}}{2^{3}}+\cdots+\frac{F_{n-1}}{2^{n-1}}\right]+\frac{F_{1}}{2^{1}}+\frac{F_{0}}{2^{0}}\right) .
$$

TABLE II

NUMBER OF THIS TYPE OF SYNTHETIC CHANNELS

\begin{tabular}{|c|c|c|}
\hline$n=\log _{2} N$ & Cardinality of the attractor set & Code rate \\
\hline 6 & 21 & 0.6719 \\
\hline 7 & 34 & 0.7344 \\
\hline 8 & 55 & 0.7852 \\
\hline 9 & 89 & 0.8262 \\
\hline 10 & 144 & 0.8594 \\
\hline 11 & 233 & 0.8862 \\
\hline 12 & 377 & 0.9080 \\
\hline 13 & 610 & 0.9255 \\
\hline 14 & 987 & 0.9398 \\
\hline 15 & 1597 & 0.9513 \\
\hline 16 & 2584 & 0.9606 \\
\hline
\end{tabular}

Here, notice that $\frac{F_{0}}{2^{0}}=0$ and $\frac{F_{1}}{2^{1}}=1 / 2$. The final exact expression is

$$
\Delta=2^{n-1} \sum_{t=0}^{n-1} F_{t} / 2^{t} .
$$

There is the power series $\sum_{t=0}^{\infty} F_{t} \cdot k^{-t}=\frac{k}{k^{2}-k-1}$ for integer $k>1$.

As a result,

$\lim _{n \rightarrow \infty} \frac{\text { Number of channels with (11) }}{\text { Number of all channels }}=\lim _{n \rightarrow \infty} \frac{2^{n} \frac{1}{2} \frac{2}{2^{2}-2-1}}{2^{n}}=1$.

Now we can consider here how we can benefit from the definition of the attractor in code design for long block lengths.

For this purpose, we consider the natural channel with LLR parameter is greater than $\pi / 2$. In this case, the synthetic channel indices to be identified by the attractor will start from the most significant bit position, and the different length sequences will be determined which will reduce the LLR value of the natural channel to less than $\pi / 2$ as an inter-level LLR value, (please see the Example 4). For more clarity, we provide the following plain text for efficient design.

Definition 5. As a plain text an efficient code design can be given as follows.

i) Define an attractor set $\Omega$ as a subset of $\{1,2, \ldots, N\}$ for $n$,

ii) for $i=1, \ldots, n$

Apply $i$-th partial order operator to update $\Omega$

end

iii) Apply simplified Gaussian approximation for the complement of the set $\Omega$

Example 5. We consider $n=6$ in this example. There are 64 synthetic channels placed in Table III. Here, black bold face binary expansions denote a channel identified by the attractor (i.e. they do not have 11). There are $F_{n+2}=21$ this type of channels for $n=6$ that are universally less reliable than the natural channel.

We apply the multiple partial order to find more channels that are worse than the natural channel $W$. When we consider the first order operator to increase the number of bad channels, there are not any new bad channel by removing 1 in 
TABLE III

EXAMPLE FOR ATtRactor AND MUltiple PARTIAL ORder

\begin{tabular}{|l|l|l|l|}
\hline $\mathbf{0 0 0 0 0 0}$ & $\mathbf{0 0 1 0 0 0}$ & $\mathbf{0 1 0 0 0 0}$ & $\mathbf{0 1 1 0 0 0}$ \\
\hline $\mathbf{0 0 0 0 0 1}$ & $\mathbf{0 0 1 0 0 1}$ & $\mathbf{0 1 0 0 0 1}$ & $\mathbf{0 1 1 0 0 1}$ \\
\hline $\mathbf{0 0 0 0 1 0}$ & $\mathbf{0 0 1 0 1 0}$ & $\mathbf{0 1 0 0 1 0}$ & $\mathbf{0 1 1 0 1 0}$ \\
\hline $\mathbf{0 0 0 0 1 1}$ & 001011 & $\mathbf{0 1 0 0 1 1}$ & 011011 \\
\hline $\mathbf{0 0 0 1 0 0}$ & $\mathbf{0 0 1 1 0 0}$ & $\mathbf{0 1 0 1 0 0}$ & $\mathbf{0 1 1 1 0 0}$ \\
\hline $\mathbf{0 0 0 1 0 1}$ & $\mathbf{0 0 1 1 0 1}$ & $\mathbf{0 1 0 1 0 1}$ & 011101 \\
\hline $\mathbf{0 0 0 1 1 0}$ & $\mathbf{0 0 1 1 1 0}$ & $\mathbf{0 1 0 1 1 0}$ & 011110 \\
\hline $\mathbf{0 0 0 1 1 1}$ & 001111 & 010111 & 011111 \\
\hline \hline $\mathbf{1 0 0 0 0 0}$ & $\mathbf{1 0 1 0 0 0}$ & 110000 & 111000 \\
\hline $\mathbf{1 0 0 0 0 1}$ & $\mathbf{1 0 1 0 0 1}$ & 110001 & 111001 \\
\hline $\mathbf{1 0 0 0 1 0}$ & $\mathbf{1 0 1 0 1 0}$ & 110010 & 111010 \\
\hline $\mathbf{1 0 0 0 1 1}$ & 101011 & 110011 & 111011 \\
\hline $\mathbf{1 0 0 1 0 0}$ & 101100 & 110100 & 111100 \\
\hline $\mathbf{1 0 0 1 0 1}$ & 101101 & 110101 & 111101 \\
\hline $\mathbf{1 0 0 1 1 0}$ & 101110 & 110110 & 111110 \\
\hline 100111 & 101111 & 110111 & 111111 \\
\hline
\end{tabular}

the attractor. The result is guaranteed that it is placed in the attractor. Then, we can apply second order operator to find more synthetic channels. For example; $W_{(101000)}$ is a member of the attractor (i.e., $01 \rightarrow 10$ ). By using 2 nd order operator, we have the following result.

$$
W_{(011000)} \prec W_{(101000)} \prec W .
$$

Finally, we can apply third order operator. For example; $W_{(011100)}$ is a member of the bad channels that are union set of attractor and 2nd order operator. By using 3rd order operator (i.e., 0110 $\rightarrow 1001$ ), we have the following result.

$$
W_{(011100)} \prec W_{(101010)} \prec W .
$$

The synthetic channels found by multiple partial order are denoted by blue bold face in Table. III.

As a result, we have found 35 synthetic channels that are worse than the natural channels for $L L R<\pi / 2$. On the other hand 25 of them is still worse than the natural channel for $L L R<\pi$ (i.e., they do not have 1 in the first bit position).

\section{CONCLUSION}

The universal features of polar codes are an important advantage for efficient code design problems. In this work, we have defined new type of universal features. We have made it easier to calculate the reliability of synthetic channels that are important for efficient code design. For this purpose, we simplified the recursive update functions of the Gaussian approximation method. Then, we have provided the attractor set of indices of a significant number of synthetic channels that are less reliable than the original channel. We also described the cardinality of the attractor set using Fibonacci numbers.

\section{APPENDIX A}

SimplificAtion of THE FUnCTIONS: $\phi(x)$ AND $\phi^{-1}(x)$

First, we consider the following assumption:

$$
\tanh x \approx\left\{\begin{array}{cc}
1, & x>0 \\
0, & x=0 \\
-1, & x<0
\end{array}\right.
$$

Then, we use the following equations.

$$
\begin{aligned}
& \frac{1}{\sqrt{4 \pi x}} \int_{-\infty}^{\infty} \tanh \frac{u}{2} e^{-\frac{(u-x)^{2}}{4 x}} d u \approx \\
& \frac{1}{\sqrt{4 \pi x}}\left(\int_{0}^{\infty} e^{-\frac{(u-x)^{2}}{4 x}} d u-\int_{-\infty}^{0} e^{-\frac{(u-x)^{2}}{4 x}} d u\right)
\end{aligned}
$$

We apply the transformation: $\frac{u-x}{2 \sqrt{x}}=v$. Then,

$$
\begin{aligned}
& \frac{1}{\sqrt{4 \pi x}}\left(\int_{0}^{\infty} e^{-\frac{(u-x)^{2}}{4 x}} d u-\int_{-\infty}^{0} e^{-\frac{(u-x)^{2}}{4 x}} d u\right)= \\
& \frac{1}{\sqrt{\pi}}\left(\int_{-\sqrt{x} / 2}^{\infty} e^{-v^{2}} d v-\int_{-\infty}^{-\sqrt{x} / 2} e^{-v^{2}} d v\right) .
\end{aligned}
$$

Then, we use the definition:

$$
\operatorname{erfc}\left(\frac{\sqrt{x}}{2}\right)=\frac{2}{\sqrt{\pi}} \int_{\sqrt{x} / 2}^{\infty} e^{-v^{2}} d v .
$$

$$
\begin{aligned}
& 1-\operatorname{erfc}\left(\frac{\sqrt{x}}{2}\right)= \\
& \frac{1}{\sqrt{4 \pi x}}\left(\int_{0}^{\infty} e^{-\frac{(u-x)^{2}}{4 x}} d u-\int_{-\infty}^{0} e^{-\frac{(u-x)^{2}}{4 x}} d u\right) .
\end{aligned}
$$

Finally, we have the simplified equations as follows:

$$
\begin{gathered}
\phi(x)=\operatorname{erfc}\left(\frac{\sqrt{x}}{2}\right), \\
\phi^{-1}(x)=4(\operatorname{erfcinv}(x))^{2} .
\end{gathered}
$$

\section{ACKNOWLEDGMENT}

This work was performed in postdoc study at Bilkent University supported by the Scientific and Technological Research Council of Turkey (TÜBİTAK), grant: 1929B011500065.

I would like to thank Prof. E. Arıkan for helpful discussions.

\section{REFERENCES}

[1] E. Arıkan, "Channel polarization: A method for constructing capacityachieving codes for symmetric binary-input memoryless channels," IEEE Trans. Inf. Theory, vol. 55, no. 7, pp. 3051-3073, Jul. 2009.

[2] R. Mori and T. Tanaka, "Performance of polar codes with the construction using density evolution," IEEE Commun. Lett., vol. 13, no. 7, pp. 519-521, Jul. 2009.

[3] I. Tal and A. Vardy, "How to construct polar codes," IEEE Trans. Inf. Theory, vol. 59, no. 10, pp. 6562-6582, Oct. 2013.

[4] P. Trifonov, "Efficient design and decoding of polar codes," IEEE Trans. Commun., vol. 60, no. 11, pp. 3221-3227, Nov. 2012.

[5] H. Vangala, E. Viterbo, and Y. Hong, "A comparative study of polar code constructions for the awgn channel," arXiv:1501.02473, Jan. 2015.

[6] C. Schürch, "A partial order for the synthesized channels of a polar code," in Proc. IEEE Int. Symp. Inform. Theory, Jul. 2016, pp. 220224.

[7] M. Bardet, V. Dragoi, A. Otmani, and J.-P. Tillich, "Algebraic properties of polar codes from a new polynomial formalism," in Proc. IEEE Int. Symp. Inform. Theory, Jul. 2016, pp. 230-234.

[8] M. Mondelli, S. H. Hassani, and R. Urbanke, "Construction of polar codes with sublinear complexity," in Proc. IEEE Int. Symp. Inform. Theory, Jun. 2017, pp. 1853-1857.

[9] — - "Construction of polar codes with sublinear complexity," arXiv:1612.05295v4, Dec. 2016.

[10] S.-Y. Chung, T. J. Richardson, and R. L. Urbanke, "Analysis of sumproduct decoding of low-density parity-check codes using a gaussian approximation," IEEE Trans. Inf. Theory, vol. 47, no. 2, Feb. 2001. 